\title{
Implementación de la Metodologia GEEMPA en la lectoescritura musical
}

\section{Implementation of the GEEMPA methodology in musical literacy}

\author{
José Leonardo Ruiz Méndez \\ Magíster en Pedagogía. Universidad Surcolombiana. Neiva. Colombia. \\ leonardo1@usco.edu.co
}

\section{Resumen}

Al comprobar la efectividad de la Metodología Flexible para el aprendizaje de la lectoescritura (GEEMPA) en niños y niñas de los grados primero y segundo de primaria pertenecientes a una institución educativa del municipio de Palermo (Huila), el autor de esta investigación decide implementar dicha metodología a la lectoescritura musical consciente de aprovechar la experiencia desarrollada en Colombia por parte de la Corporación Conprende y el Ministerio de Educación Nacional, como un aporte significativo para la didáctica de la gramática de este lenguaje.

Palabras claves: lectoescritura musical, metodología geempa, didáctica musical, educación musical.

\section{Abstract}

By verifying the effectiveness of the flexible methodology for literacy learning (GEEMPA) in boys and girls from first and second grade in primary, belonging to an educational institution of the municipality of Palermo (Huila), the author of this research decides to implement said methodology to the musical literacy, aware of taking advantage of the experience developed in Colombia on the part of Corporación Comprender and the Ministry of National Education, as a meaningful contribution for the didactics of the grammar of this language.

Keywords: Musical literacy, GEEMPA methodology, musical didactics, musical education.

\section{¿Qué quiere decir GEEMPA?}

La metodología GEEMPA, fue creada por la Doctora Esther Pillar Grossi investigadora brasilera, alumna del sicólogo suizo Jean Piaget uno de los educadores más destacados de nuestro tiempo. Grossi, es considerada como la sucesora del filósofo y gran teórico de la educación, Paulo Freire. Matemática de profesión, se ha convertido en un símbolo latinoamericano de innovación-creación y un referente en la solución de problemas de enseñanza pública.

En 1970, Grossi creó en Porto Alegre, junto con medio centenar de profesores, el "Grupo de estudios sobre educación, metodología de investigación y acción" (GEEMPA, por sus siglas en portugués), con quienes construyó en 1982 el método del mismo nombre el cual revolucionó la educación pública en Brasil. En Colombia el Ministerio de Educación Nacional a través de la Corporación Comprende, ha implementado con éxito el método desde el año 2005.
Esta didáctica pretende garantizar el acceso al mundo letrado de niños, niñas, jóvenes y adultos que habían sido excluidos del sistema educativo por sus condiciones cognitivas. La metodología se orienta conceptualmente en la propuesta pedagógica postconstructivista: las estructuras mentales piagetianas, el aprendizaje sociocultural de Vigotzky, el concepto de intersubjetividad de Wallon y los aportes sobre Psicogénesis de la lectura y la escritura de Emilia Ferreiro, Ana Teberosky, y Sara Pain.

La didáctica GEEMPA se vale esencialmente de la lúdica como estrategia para cautivar el interés de los estudiantes y con ello garantizar más allá de la escritura y la lectura automática o memorística, procesos de producción escrita, lectura comprensiva, pensamiento lógico matemático y convivencia pacífica. 


\section{¿Por qué transversalizar el método GEEMPA con la lectoescritura musical?}

En la implementación de procesos de enseñanza aprendizaje de la música se presenta, así sea en un número reducido, un grupo de estudiantes a quienes se les dificulta el aprendizaje de la lectoescritura musical, y entonces algunos docentes comienzan a considerarlos como "faltos de talento" incluso a la hora de cantar como "desafinados". Esta dificultad trae consigo la exclusión del grupo y la promulgación de la afirmación "se nace musical" para quienes no presentan este tipo de problemas.

La anterior situación, evidencia una posible discriminación, la cual podría hacer pensar que "en nuestra cultura, la música no es indispensable, que es posible prescindir de su conocimiento, de su práctica y comprensión" (Jorquera S/F) lo que no puede ser aceptado por el fin mismo que tiene la educación de enfocarse en el ser, en sus sentimientos, en la esencia de la vida.

De esta manera, si un estudiante presenta desafinación es debido a la falta de construcción de la imagen sonora de las notas que hacen parte del tema musical que quiere interpretar. En este sentido, es necesario desarrollar en los estudiantes la construcción de un banco sonoro personal que remedie dicha situación.

Esto plantea la necesidad de crear un método de enseñanza aprendizaje de la lectoescritura musical que logre la construcción de dicha imagen sonora y por tanto la superación de las dificultades que impiden el manejo de la gramática musical en su fase inicial, entre las cuales se encuentran:

- Falta de reconocimiento de las figuras de duración

- Falta de reconocimiento de las notas en clave de sol

- Debilidad en el reconocimiento del concepto de tiempo y compás

- Desafinación de las notas de la escala natural

- Falta de interpretación de un instrumento musical afinado

Teniendo en cuenta lo anterior, este proyecto de investigación se enfoca en la implementación de la metodología GEEMPA a los procesos de enseñanza aprendizaje de la lectoescritura musical de los estudiantes matriculados en el curso Elementos de la Música Escolar correspondiente al primer semestre de la Licenciatura en Educación Artística y Cultural de la Universidad Surcolombiana.

\section{Marco Teórico}

Para comprender la importancia de la educación musical en el desarrollo integral del ser humano, es necesario tener en cuenta sus principios y objetivos, así como la historia de su implementación durante el siglo XX. De igual forma, por el contexto de la investigación se incluye el referente titulado Teoría Psicogenética de Piaget.

\section{Principios de la Educación Musical}

Los principios de la educación musical de acuerdo con lo mencionado por Pilar Pascual (2002), Profesora de la Escuela Universitaria ESCUNI adscrita a la Universidad Complutense, son los siguientes:

Valor educativo de la música: La finalidad es la formación integral de todas las facultades del hombre (psicológicas, sociológicas, psicomotoras e intelectuales), no sólo las musicales. Este principio, muestra el definitivo aporte que realiza la música al desarrollo del ser humano, por cuanto a través de ejercicios y experiencias sonoras lo equipara de herramientas que le permiten desarrollar el mundo interior y espiritual con el fin de interpretar, explicar y crear en el mundo físico que habita.

Para todos: No se trata de hacer músicos sino personas que amen la música y sepan valorarla. Con esto, se realiza una invitación a la edificación del gusto por los tipos de música que desarrollen en su máximo esplendor, los elementos que la componen: melodía, armonía y ritmo.

Libertad v Creatividad: Prima la espontaneidad, la creación y la participación. La música, a través de la exploración del mundo sonoro externo, aporta al desarrollo del ser humano la necesaria capacidad de comprender los sonidos que lo rodean con el propósito de construir y enriquecer el mundo sonoro interno que tiene cada individuo.

Progresión: la educación musical debe acompañar al ser humano en todo el proceso evolutivo. Por cuanto ella, contribuye a la construcción de la sensibilización, la capacidad de percepción, el desarrollo del movimiento, el reconocimiento espacial numérico y de conceptos.

Activo: Enseñar música debe ser transmitir el lenguaje musical en forma viva o, dicho en otros términos, aprender música haciendo música. Hacer música, es sin lugar a dudas el elemento clave para el desarrollo del proceso de enseñanza aprendizaje, pues a través de la interpretación instrumental y vocal de temas se pueden analizar los aspectos históricos, de forma y contenido estructural y de análisis de los elementos constitutivos de la música.

Lúdica: Se trata de jugar con la música. El juego es un componente vivo presente en el ser humano; esta capacidad por construir convivencia a través de él, permite la construcción del conocimiento y el desarrollo del gusto por la música.

Global: Se relaciona con otras áreas artísticas. Un buen ejemplo para entender la relación del arte con la vida misma, se encuentra en el cine. En esta manifestación 
artística, se construye una historia real o ficticia que, contada con creatividad, logra transportar al espectador al pasado o al futuro. Para contar dicha historia, se recurre a la imagen generada en escenografías, a la fotografía de espacios cerrados o abiertos (el arte visual), a la música a través de la banda sonora (el arte auditivo), a la actuación para dar vida a los personajes (el arte del cuerpo, el gesto y el movimiento); todos las artes reunidas en una, interactuando y cumpliendo la función social de comunicar.

Variedad: Debe incluir diversos aspectos (el canto, los instrumentos, el movimiento y la danza, el juego dramático, la audición, el lenguaje musical, entre otros). Un principio importante que invita a la integralidad de la educación, con el fin de lograr la integración de las artes al servicio de la aplicación de la teoría de las inteligencias múltiples de Howard Gardner, quien expone un nuevo concepto de inteligencia abordado a partir de las capacidades individuales.

Estos ocho principios, evidencian por un lado, las formas como la música se interrelaciona con las mismas artes $y$, por el otro, las posibilidades de aplicación.

\section{Objetivos de la Educación Musical}

Bukofer (citado por Pascual, 2002) menciona que hay dos objetivos en la educación musical: educación por la música y educación para la música.

Educación por la música: Persigue la comprensión y la respuesta inteligente, que facilite la amplia experiencia artística, al tiempo que la agudización de los sentidos y la estimación de los valores culturales en general. Importante objetivo que busca la noble tarea de contribuir a la evolución de los sentidos del ser humano, se pretende entonces, agudizar la percepción y desarrollar por tanto, la sensibilidad, apreciación y admiración de la belleza expresada en sonido y silencio

Educación para la música: Opera en la práctica profesional y se enfoca en la capacitación para tres campos: el compositor, el intérprete virtuoso y el musicólogo. Se intenta entonces contribuir a la realización, expresión y análisis de obras musicales. Este enfoque educativo se desarrolla en personas, que una vez sensibilizadas con el hecho sonoro, desean convertir la música en un proyecto de vida al servicio de la humanidad, de esta manera, esta persona se transforma en instrumento que contribuye al desarrollo del primer objetivo planteado.

\section{La Educación Musical en el siglo XX}

La maestra Violeta Hemsy de Gainza en el año 2000 presentó, en el marco del Primer Seminario Argentino sobre "El Modelo Artístico en la Educación Musical" (FLADEM-AR), un recuento histórico de los desarrollos pedagógicos en el campo de la Educación Musical que se sucedieron a lo largo del siglo XX y que organizó en períodos y categorías así:

\section{Primer período (1930-1940).}

\section{De los métodos precursores}

Entre los enfoques precursores se cuentan el método denominado "Tonic-Sol-Fa" 2 en Inglaterra ("TonikaDo" en Alemania), y el método de Maurice Chevais en Francia, el cual -entre otros recursos- utiliza la fonomimia en la didáctica del canto en el nivel inicial. Respecto del método "Tonic-Sol-Fa".

Destaca que en este período de tiempo se inició en Europa el movimiento pedagógico denominado "Escuela nueva" o "Escuela activa", la cual desatacaba la personalidad y las necesidades del educando. Los métodos "activos"-Pestalozzi, Decroly, Froebel, Dalton, Montessori- se difunden en Europa y Norteamérica e influencian posteriormente la educación musical en América Latina.

Segundo período (1940-1950).

De los métodos activos

Entre las figuras sobresalientes de la pedagogía musical de los países europeos que ejercen su influencia en este período se destaca, por su acción vanguardista, el músico y educador suizo $\mathrm{E}$. Jacques Dalcroze (1865-1950), creador de la Euritmia. El panorama pedagógico se enriquece más tarde con los aportes personalísimos de Edgar Willems (1890 1978, Bélgica-Suiza) y Maurice Martenot (1898-1980, Francia); ambos ratificarán oportunamente sus coincidencias conceptuales básicas con J. Dalcroze, en relación a la educación musical.

\section{Tercer período (1950-1960).}

\section{De los métodos instrumentales}

Se incluye en esta la categoría, los métodos del alemán Carl Orff (1895-1982), centrado en los conjuntos instrumentales; del húngaro Zoltán Kodály (18821967), que privilegia la voz y el trabajo coral, y del japonés Suzuki (1898-1998), que inicialmente se focaliza en la enseñanza del violín.

Orff dará ahora prioridad a la producción de piezas y materiales orientados a estimular la ejecución grupal (instrumental, vocal, corporal). Este compositor produce una obra didáctica en cinco tomos (el "Orff Schulwerk") que integra los juegos lingüísticos y el movimiento corporal al conjunto vocal-instrumental. En el mundo occidental se multiplican los grupos de percusión a base del "instrumental Orff", y se ejecutan y difunden las alegres piezas para niños y jóvenes del Orff Schulwerk. Con Kodály, en cambio, aprendimos a valorar el folclore, objeto imprescindible de la educación musical. La Maestra Hemsy de Gainza y el maestro Guillermo Graetzer publicaron los cancioneros "Canten señores cantores" y "Canten señores cantores de América" motivados por el aporte de Kodaly a la educación musical latinoamericana.

Cuarto período (1970-1980). De los métodos creativos El aporte de la llamada "generación de los composi- 
tores" (G. Self, B. Dennis, J. Paynter, M. Schafer, etc.), a la cual nos hemos referido en detalle en otras obras, marca con su influencia la educación musical de las décadas del 70 y 80 .

Ouinto Deríodo (1980-1990). De transición

La diversidad de las problemáticas musicales y pedagógicas contribuye, en este período, a desdibujar los contornos de la educación musical, en tanto objeto de conocimiento. Continúa el interés por la música contemporánea en el aula, pero al mismo tiempo el campo educativo-musical recibe el influjo de numerosas tendencias: la tecnología musical y educativa, la ecología, los movimientos alternativos en el arte, la nueva corporalidad, la musicoterapia, las técnicas grupales, etcétera.

\section{Sexto período (1990). De los nuevos paradigmas}

Los educadores musicales tienen a su disposición una diversidad de modelos. En la pedagogía musical europea (sobre todo en España) está en boga la enseñanza-aprendizaje de juegos rítmico-corporales y danzas étnicas (especialmente de origen africano) y populares. En clases generalmente nutridas, los alumnos practican en grupo, en forma entusiasta, patrones sonoros y de movimiento a veces sumamente complejos (modelo étnico, paradigma recreativo, de acción: aprender haciendo). En algunos países de Latinoamérica niños y jóvenes aprenden música a través de diversas prácticas populares (canto, danzas, bandas, juegos, ceremonias, etc.) que incluyen la participación corporal y la actuación. Algunos docentes prefieren o se identifican más con los modelos ecológicos (por ejemplo, el enfoque de Murray Schafer), lúdicos, tecnológicos, etc.

\section{Teoría Psicogenética de Piaget}

A Jean Piaget le interesó el estudio del desarrollo del conocimiento humano desde la infancia hasta la adolescencia. Su pregunta de investigación inicial fue ¿Cuál es la naturaleza y el origen del conocimiento humano? En otras palabras ¿cómo llega el ser humano a desarrollar sus procesos de cognición?

Para comprender la dimensión de la pregunta, es necesario definir primero ¿Qué es el conocimiento o la cognición?

Según Martín (2000) el término cognición "comprende toda una serie de procesos mentales que realizan los seres humanos para adquirir, retener, interpretar, comprender, organizar, utilizar y reutilizar tanto la información existente en el medio que nos rodea, como la propia información ya adquirida y almacenada".

Pero... ¿Por qué es importante estudiar la cognición? Porque la cognición incluye los procesos de percepción, atención, cognición espacial, imaginación, lenguaje, memoria, resolución de problemas, creatividad, pensamiento e inteligencia, aspectos fundamentales que el ser humano necesita desarrollar para aprender, entender la realidad y crear conocimiento.
Entonces, ¿Por qué es necesario relacionar aprendizaje, conocimiento y realidad?. De la misma manera como al interior del cuerpo humano se llevan a cabo los procesos biológicos como respirar, comer, dormir, los cuales utilizamos para adaptarnos y lograr sobrevivir a un mundo en permanente cambio, así los procesos cognitivos como la memoria, la resolución de problemas y la inteligencia se llevan a cabo al interior de nuestro organismo para llegar al mismo sentido adaptativo. En esto consiste la teoría organísmica, representada por autores como Heinz Werner (1957) y muy especial-mente Piaget y colaboradores.

Teniendo en cuenta lo anterior, entonces ¿qué es aprendizaje?. Según Ignacio Pozo (1996) el aprendizaje consiste en el progreso de las estructuras cognitivas mediante procesos de equilibración, de modo que el aprendizaje se produce cuando se da un desequilibrio o un conflicto cognitivo entre estos dos procesos de construcción estática del conocimiento (en el sentido de que la nueva información se asimila a las estructu-ras de conocimiento ya existentes) y la construcción dinámica o, en términos piagetianos, acomodación, que es verdaderamente la que permite mediante determinados procesos modificar y 'construir' el conocimiento.

Entonces, para alcanzar el aprendizaje, ¿Por qué es necesario provocar un desequilibrio o un conflicto cognitivo? Porque al interior de nuestro cerebro se llevan a cabo dos procesos: asimilación y acomodación, los cuales muestran la evolución intelectual del individuo.

El desequilibrio que se encuentra se alcanza en la medida en que se asimila y se acomoda el conocimiento. Piaget estableció tres niveles de este proceso de equilibración:

- El equilibrio se establece entre los esquemas del sujeto y los acontecimientos externos.

- El equilibrio se establece entre los propios esquemas del sujeto

- El equilibrio se traduce en una integración jerárquica de esquemas diferenciados.

Pero, ¿En qué momento aparece el Conflicto Cognitivo? Cuando entran en contradicción los esquemas internos con los esquemas externos. De esta manera se rompe el equilibrio cognitivo y el ser humano se plantea interrogantes, investiga, descubre,...etc., busca respuestas, hasta llegar al conocimiento que le permita volver de nuevo al equilibrio cognitivo.

Volvamos a la pregunta inicial ¿Cuál es la naturaleza y el origen del conocimiento humano? Piaget encontró cuatro periodos de desarrollo cognitivo:

\footnotetext{
Etapa Sensoriomotora: 0 a 24 meses

- Etapa Preoperacional: 2 a 7 años

- Etapa de las operaciones concretas: 7 a 11 años

- Etapa de las operaciones formales: 11 años en adelante
} 
Entonces, ¿El proceso de cognición termina en la Etapa de las operaciones Formales, según Piaget?. Inicialmente Piaget había llegado a la conclusión que el crecimiento físico y psicológico individual terminaba en un estadio final de desarrollo, en este caso en la madurez biológica que se alcanza en la adolescencia, por cuanto se tendría desarrollado el pensamiento abstracto que se relaciona con la Etapa de las operaciones formales.

Sin embargo, Piaget realiza una revisión a su teoría, motivado por investigadores que retomaban continuamente sus estudios, y establece que entre los 15 y los 20 años de edad se efectúa la plena aparición de las operaciones formales teniendo en cuenta la riqueza de la estimulación cognitiva que alcanzó el individuo en su infancia (Clemente, 1996).

¿Qué ha pasado luego de las conclusiones del estudio que realizó Piaget?. Autores como Peter D. Kramer (1983), Michael Commons, Francis Richards y Cheryl Armon (1984); John Rybash, William Hoyer y Paul Roodin (1986); Patricia Arlin (1975, 1984) entre otros han propuesto una nueva etapa de desarrollo del conocimiento denominada "De las operaciones postformales" la cual se encuentra enfocada en los adultos.

Esta etapa de operaciones postformales tiene las siguientes características básicas:

\section{-Pensamiento divergente y creativo:}

Acepta la contradicción como un aspecto importante en la comprensión de la realidad. Se encuentra aquí que la naturaleza del pensamiento es relativo. Arlin (citada por Martín, 2000) argumenta que el desarrollo del pensamiento postformal implica el descubrimiento de nuevos problemas que no tienen una única solución, sino varias, en las cuales se consideran elementos no solo cognitivos sino emocionales de los que se seleccionan las más realistas, las más adaptadas al contexto, las más originales (pensamiento creativo).

\section{-Pensamiento metasistemático:}

Rybash, Hoyer \& Armon (citados por Martín, 2000) mencionan que si un individuo puede observar la realidad en términos no sólo lógicos, sino relativos y contradictorios, entonces esta realidad debe estar configurada como una estructura que adopta la forma de sistema abierto y dinámico, constituido por múltiples dimensiones que interactúan entre sí, lo que permite a los adultos contemplarla en su globalidad, de modo integral y completo.

\section{-Pensamiento Dialéctico}

Martín (2000) menciona que Klaus Riegel en 1976 realizó una crítica a la teoría de Piaget a través de su "Manifiesto Dialéctico" en el cual abogaba por abandonar y sustituir esta teoría basada en el equilibrio y la estabilidad por otro planteamiento basado en el desequilibrio y el cambio, en el cual pone de relieve la existencia de un conflicto permanente entre fuerzas interactivas en continuo cambio. El conflicto se aborda desde tres perspectivas: Idea (tesis) opuesto (antítesis) y resolución (síntesis).

\section{- Pensamiento Contextualizado}

Martín (2000) señala que el pensador postformal adulto no puede guiarse exclusivamente por principios universales, rígidos, lógicos y absolutos, dada la naturaleza en permanente cambio de la vida cotidiana y real. De este modo, el adulto crea nuevos principios basados en las circunstancias cambiantes y específicas de su vida.

\section{Metodología}

La metodología que desarrolla la presente investigación se fundamenta en la propuesta elaborada por la Dra. Grossi y su grupo de colaboradores, la cual denominaron "Metodología GEEMPA para la lectoescritura"

Este estudio se basa en la investigación acción por cuanto los sujetos de investigación son los protagonistas durante el proceso de construcción del conocimiento.

\section{Definición del Universo}

El Programa de Licenciatura en Educación Artística y Cultural es un programa académico ofrecido por la Facultad de Educación de la Universidad Surcolombiana, institución ubicada en la ciudad de Neiva, departamento del Huila. En la actualidad el programa mencionado cuenta con 163 estudiantes matriculados, de los cuales 45 hacen parte de la cohorte 2014 .

La Licenciatura en su plan de estudios, cuenta con cuatro áreas de formación a saber: Artística, Historia y Teoría del Arte, Pedagógica y Desarrollo Humano. El área de Formación Artística se encuentra a su vez conformada por tres subáreas: escénica, musical y visual.

La subárea musical se encuentra integrada por siete cursos: Elementos de la Música Escolar, Elementos e Instrumentos de la Música Regional, Elementos e Instrumentos de la Música Colombiana, Elementos e Instrumentos de la Música Latinoamericana, Elementos e Instrumentos de la Música Universal, Elementos e Instrumentos de la Música Contemporánea, Elementos de Dirección Musical.

El curso de Elementos de la música Escolar, que se implementa en el primer semestre de la carrera, tiene un total de 3 créditos académicos los cuales corresponden a 144 horas semestrales distribuidas de la siguiente manera: 64 horas de trabajo presencial y 80 horas de trabajo independiente.

\section{Sujetos de Investigación}

Del total de estudiantes matriculados en la Licenciatura (163), la investigación define como base de sondeo los 45 estudiantes inscritos en el curso 
Elementos de la Música Escolar que corresponde al primero de los cursos de la subárea de Formación Musical.

Se selecciona esta población, primero, porque se constituye en población de fácil acceso debido a la relación laboral existente entre el investigador y institución educativa a la cual se encuentra vinculada la población, y, segundo, porque se espera que el trabajo propuesto se retroalimente continuamente con los grupos de primer semestre de la subárea musical.
El grupo de estudiantes está conformado por 18 mujeres y 28 hombres los cuales tienen una edad promedio de 20 años. En su mayoría se encuentra ubicados en el Estrato Social 2 y sus lugares de procedencia son: del Departamento del Huila proceden de los municipios de Garzón, Pitalito, Rivera, La Plata, La Argentina, Guadalupe, Tarqui, Altamira, Palermo, Yaguará, Gigante, Saladoblanco, Oporapa, Guadalupe y Neiva. De otros Departamentos: Cundinamarca, Bogotá; Cauca, Páez; Cesar, Manaure Balcón del Cesar; Valle del Cauca, Cali; y, Putumayo, Puerto Asís. (Información tomada de la base de datos de la Universidad Surcolombiana www.usco.edu.co ).

\section{Enfoque Metodológico}

En la siguiente tabla se presenta el Enfoque Metodológico de la presente investigación:

\begin{tabular}{|c|c|c|c|c|}
\hline VARIABLES & INDICADORES & FUENTES & TÉCNICAS & INSTRUMENTOS \\
\hline Conocimiento Previo & Conocimiento Previo & Estudiantes & Entrevista & $\begin{array}{l}\text { Acta de Compromiso } \\
\text { Académico } \\
\text { Clase Entrevista }\end{array}$ \\
\hline \multirow[t]{6}{*}{ Ritmo } & Nivel Rítmico 0 & \multirow[t]{6}{*}{ Estudiantes } & \multirow[t]{6}{*}{ Taller } & \multirow{6}{*}{$\begin{array}{l}\text { Prueba No.1 de Nivel Rítmico } \\
\text { Prueba No.2 de Nivel Rítmico } \\
\text { Prueba No.3 de Nivel Rítmico }\end{array}$} \\
\hline & Nivel Pre-Rítmico 1 & & & \\
\hline & Nivel Pre-Rítmico 2 & & & \\
\hline & Nivel Rítmico 1 & & & \\
\hline & Nivel Rítmico 2 & & & \\
\hline & Nivel Rítmico 3 & & & \\
\hline \multirow[t]{6}{*}{ Melodía } & Nivel Melódico 0 & \multirow[t]{6}{*}{ Estudiantes } & \multirow[t]{6}{*}{ Taller } & \multirow{6}{*}{$\begin{array}{l}\text { Prueba No.1 de Nivel } \\
\text { Melódico } \\
\text { Prueba No.2 de Nivel } \\
\text { Melódico } \\
\text { Prueba No.3 de Nivel } \\
\text { Melódico }\end{array}$} \\
\hline & Nivel Pre-melódico 1 & & & \\
\hline & Nivel Pre-melódico 2 & & & \\
\hline & Nivel Melódico 1 & & & \\
\hline & Nivel Melódico 2 & & & \\
\hline & Nivel Melódico 3 & & & \\
\hline
\end{tabular}

\section{Variables}

- Conocimiento Previo:

Esta variable hace referencia a la necesidad que encontró el investigador de aplicar una prueba escrita mediante la cual se pueda dar una valoración de entrada al grupo respecto al conocimiento previo que tiene acerca de las figuras de duración, el pentagrama, su lectura y escritura.

- Ritmo:

Es uno de los Elementos de la Música. El estudio del ritmo en la gramática musical está representado por las figuras de duración, el manejo del compás así como del tempo.

- Melodía

Es el segundo elemento constitutivo de la Música. Para este proyecto de investigación representa el dominio de las figuras de duración en el pentagrama en lo referente a su lectura y escritura.

\section{Indicadores}

Es importante mencionar que cada uno de los estudiantes, de acuerdo con las respuestas obtenidas en las pruebas diseñadas, será ubicado en cada uno de los mismos. De esta manera, los estudiantes procurarán mejorar en los resultados de las diferentes pruebas, con el fin de lograr una ubicación superior.

Variable Ritmo:

- Nivel Rítmico 0

No hay reconocimiento de las figuras de duración en cuanto a su escritura y lectura.

- Nivel Pre-Rítmico 1

Existe un mínimo reconocimiento de las figuras de duración sin que haya claridad en el reconocimiento de las células rítmicas trabajadas. El estudiante presenta una dificultad importante en la escritura y lectura de los signos musicales. Reconocimiento de menos de 5 células rítmicas trabajadas. 
- Nivel Pre-Rítmico 2

El estudiante ha alcanzado algún reconocimiento de las figuras de duración. Existe un mejoramiento mínimo en la escritura de las figuras de duración en cuanto a su elaboración pero persisten los problemas de escritura y lectura. Reconocimiento de menos de 10 células rítmicas trabajadas.

- Nivel Rítmico 1

El estudiante ha superado los niveles iniciales de escritura musical, sin embargo presenta dificultad en el reconocimiento de algunas de las células rítmicas trabajadas en los talleres programados. Reconocimiento entre 10 y 13 células rítmicas trabajadas.

- Nivel Rítmico 2

El estudiante reconoce las células rítmicas trabajadas en las fichas elaboradas para tal fin. La representación gráfica de los signos musicales es clara. Reconocimiento total de las 14 células rítmicas trabajadas.

- Nivel Rítmico 3

Es el nivel más alto de la escala de valor rítmico. El estudiante reconoce las células rítmicas en un orden diferente al evaluado en el Nivel Rítmico 1 y el Nivel Rítmico 2.

Variable Melodía:

- Nivel Melódico 0

No hay reconocimiento del pentagrama en clave de sol.

- Nivel Pre-Melódico 1

Existe un mínimo reconocimiento del pentagrama en clave de sol. Existe confusión en la relación cifrado americano y posición de las notas en el pentagrama en clave de sol. Presenta dificultad en la escritura de las figuras de duración en el pentagrama, como por ejemplo la ubicación correcta en la línea o en el espacio. Escribe menos de 5 compases de los ejercitados en los talleres.

- Nivel Pre-Melódico 2

El estudiante ha alcanzado algún reconocimiento de la ubicación de las notas musicales en el pentagrama en clave de sol, sin embargo presenta dudas en la lectura y confusión en la ubicación de la plica. El estudiante logra realizar lectura y escritura de menos de 10 compases de los ejercitados en los talleres.

- Nivel Melódico 1

El estudiante ha superado los niveles iniciales de lectura musical en el pentagrama sin embargo falta más seguridad en el reconocimiento de algunas de las notas en el pentagrama. Escribe correctamente menos de 18 compases de los definidos en los talleres de trabajo.
- Nivel Melódico 2

El estudiante reconoce a plenitud los 18 compases definidos y trabajados en los talleres. La representación gráfica de los signos musicales es clara.

- Nivel Melódico 3

Es el nivel más alto de la escala de valor melódico. El estudiante reconoce las notas musicales ubicadas en el pentagrama en clave de sol en un orden diferente al evaluado en el Nivel Melódico 1 y el Nivel Melódico 2.

\section{Instrumentos}

Los instrumentos definidos para la presente investigación son: Acta de Compromiso académico, Clase Entrevista, Pruebas de Nivel Rítmico 1,2 y 3, Prueba de Nivel Melódico 1,2 y 3.

\section{Acta de Compromiso Académico}

A diferencia del Método GEFMPA, para este proyecto se utiliza el nombre de Acta de Compromiso en lugar de Contrato Didáctico. Este es un documento elaborado entre estudiantes y profesores que contiene claramente los compromisos de las dos partes, como la asistencia, la puntualidad, la colaboración y todo lo que el docente desee incluir sobre pautas para el desarrollo de la didáctica y sus obligaciones. Se aplica en la primera semana del primer mes.

\section{Clase Entrevista}

El formato diseñado contiene siete preguntas las cuales pretenden indagar acerca de los conocimientos previos que tienen los estudiantes inscritos al curso de Elementos de la Música Escolar, el cual se desarrolla en el primer semestre de la Licenciatura en Educación Artística y Cultural de la Universidad Surcolombiana. Se aplica en la primera semana del primer mes.

Los aspectos tenidos en cuenta para esta valoración son los siguientes:

- Escritura de célula rítmica dictada

- Lectura de célula rítmica dada

- Escritura en el pentagrama de frase musical dada

- Lectura de partitura dada (con presencia de diversos signos musicales)

- Lectura de Frase musical dada (reconocimiento de líneas y espacios del pentagrama así como la lectura de figuras de duración)

- Escritura de signos musicales conocidos por el estudiante

- Escritura del nombre de los signos musicales dados.

\section{Prueba No.1 Nivel Rítmico}

Se realiza en la tercera semana. Evalúa el nivel de comprensión del proceso de aprendizaje de las 14 células rítmicas trabajadas en los talleres No. 1 y No.2. 


\section{Prueba No. 2 Nivel Rítmico}

Se realiza en la sexta semana. Evalúa el nivel de comprensión del proceso de aprendizaje de las 14 células rítmicas trabajadas en los talleres No. 1, No.2, No.3, No. 4 y No. 5.

\section{Prueba No. 3 Nivel Rítmico}

Se realiza en la novena semana. Evalúa el nivel de comprensión del proceso de aprendizaje de las 14 células rítmicas trabajadas en los talleres siete (7) talleres del Nivel Rítmico.

\section{Prueba No. 1 Nivel Melódico}

Se realiza en la décimo segunda semana. Evalúa el nivel de comprensión del proceso de aprendizaje de las 18 células rítmico melódicas trabajadas en los talleres No. 8 yNo.9.

\section{Prueba No. 2 Nivel Melódico}

Se realiza en la décimo cuarta semana. Evalúa el nivel de comprensión del proceso de aprendizaje de las 18 células rítmico melódicas trabajadas en los talleres No. 10 y No.11.

\section{Prueba No. 3 Nivel Melódico}

Se realiza en la décimo sexta semana. Evalúa el nivel de comprensión del proceso de aprendizaje de las 18 células rítmico melódicas trabajadas en los talleres No. 12 y No.13.

\section{Resultados}

Referente a las tres pruebas diseñadas para el Nivel Rítmico se encontraron los siguientes resultados finales teniendo en cuenta los indicadores definidos para la presente investigación:

\section{INDICADORES}

Nivel Rítmico Cero

Nivel Pre-Rítmico 1

Nivel Pre-Rítmico 2

Nivel Rítmico 1

Nivel Rítmico 2

Nivel Rítmico 3

El 71\% del grupo alcanzó en la Prueba No. 3 del Nivel Rítmico, los Niveles Rítmico 1,2 y 3 lo que contrasta con el $100 \%$ de estudiantes que mostró en la Clase Entrevista (aplicada en la primera semana), no tener desarrollada la habilidad para representar gráficamente las células rítmicas dictadas. Por su parte, el 29\% del grupo presenta alguna dificultad en el reconocimiento (lectura y escritura) de las células rítmicas trabajadas en los talleres.

Este mismo porcentaje del 71\% logrado luego de la aplicación de la Prueba No.3 del Nivel Rítmico, duplica el resultado obtenido alcanzado en la Clase Entrevista para la ejecución correcta de las células rítmicas dadas.

En lo referente al reconocimiento y escritura de signos musicales, el 71\% alcanzado luego de la aplicación de la Prueba No.3 del Nivel Rítmico, contrasta con el $24 \%$ y el $35 \%$ respectivamente, hallado en la Clase Entrevista

En la siguiente tabla se muestra el consolidado de los resultados alcanzados luego de la aplicación de las Prueba No.1, No.2 y No.3 en los diferentes Indicadores Nivel Cero, Nivel Premelódico 1, Nivel Premelódico 2, Nivel Rítmico 1, Nivel Rítmico 2, Nivel Rítmico 3:

$\begin{array}{lccc}\text { INDICADORES } & \begin{array}{c}\text { PRUEBA } \\ \text { No. 1 }\end{array} & \begin{array}{c}\text { PRUEBA } \\ \text { No. 2 }\end{array} & \begin{array}{c}\text { PRUEBA } \\ \text { No. 3 }\end{array} \\ \text { Nivel Melódico Cero } & 2 \% & 0 \% & 0 \% \\ \text { Nivel Pre-Melódico 1 } & 34 \% & 39 \% & 5 \% \\ \text { Nivel Pre-Melódico 2 } & 17 \% & 11 \% & 15 \% \\ \text { Nivel Melódico 1 } & 27 \% & 27 \% & 20 \% \\ \text { Nivel Melódico 2 } & 20 \% & 23 \% & 28 \% \\ \text { Nivel Melódico 3 } & 0 \% & 0 \% & 32 \%\end{array}$

El 80\% del grupo, luego de la aplicación de la Prueba No.3 del Nivel Melódico, logró los Niveles 1,2,3; esto contrasta con los resultados obtenidos en la Clase Entrevista (escritura musical en el pentagrama) que tan solo presentó un $13 \%$ de efectividad en la respuesta, así como el $24 \%$ obtenido en la lectura de una frase musical dada.

\section{Conclusiones}

La implementación de la metodología GEEMPA propuesta por la Dra. Grossi a la lectoescritura musical, abordada en este proyecto de investigación, permitió comprobar la bondad y eficacia de este método a través del cual se logró dar inicio al proceso de formación musical de los estudiantes de primer semestre de la Licenciatura en Educación Artística y Cultural de la Universidad Surcolombiana.

Este comienzo, estuvo trasversalizado por la acción lúdica que pretende el método original a través del cual se logró comprobar lo siguiente:

1. En cuanto a la firma del Acta de Compromiso académico (Contrato Didáctico): Esta fue una experiencia importante para el grupo de estudiantes pues les permitió conocer y aceptar sus compromisos.

2. La Clase Entrevista: permitió llevar acabo el encuentro académico entre docente y estudiante a partir del cual se pudo establecer el conocimiento previo respecto de la lectoescritura musical.

3. Contexto Musical: El autor de la investigación realizó la composición del tema musical "Es mi Can" el cual se convirtió en la situación significativa para el grupo de estudiantes. Cada uno de los talleres diseñados tiene en cuenta desde el punto de vista rítmico y melódico la composición mencionada. 
4. Elaboración de fichas didácticas: Se diseñaron 13 talleres, que incluyeron diversos juegos con las células rítmicas y melódicas del tema musical "Es mi Can" Las fichas didácticas diseñadas se utilizaron en el juego Concéntrese y Veritek Rítmico y melódico, así como Crucigrama Rítmico y Melódico.

5. Carpeta: Cada estudiante tuvo la responsabilidad de organizar su carpeta donde se archivaban los talleres resueltos por ellos mismos.

6. Organización de grupos según la Psicogénesis: Para el desarrollo del proyecto de investigación, el autor del mismo, asignó el nombre de indicadores a saber:

Variable Ritmo:

- Nivel Rítmico 0

- Nivel Pre-Rítmico 1

- Nivel Pre-Rítmico 2

- Nivel Rítmico 1

- Nivel Rítmico 2

- Nivel Rítmico 3

Variable Melodía:

- Nivel Melódico 0

- Nivel Pre-Melódico 1

- Nivel Pre-Melódico 2

- Nivel Melódico 1

- Nivel Melódico 2

- Nivel Melódico 3

Luego de la aplicación de cada una de las pruebas diseñadas, se proyectó en video beam los resultados de las mismas, a través de las cuales los estudiantes conocieron su ubicación en la escala prevista teniendo en cuenta los indicadores antes enunciados.

Al respecto, los estudiantes se mostraron motivados no solo por conocer el avance en su proceso de aprendizaje de la lectoescritura musical sino de mejorar en la escala de Indicadores prevista hasta alcanzar el máximo nivel.

Teniendo en cuenta todo lo anterior, se concluye que el grado de efectividad encontrado en la implementación de la metodología GEEMPA a la lectoescritura musical es del $80 \%$ teniendo en cuenta que los estudiantes vivenciaron, a través del juego, el ritmo y la melodía como elementos constitutivos de la música.

Otras conclusiones de esta investigación se presentan a continuación:

-Los resultados obtenidos en este proyecto de investigación, coinciden con lo enunciado por Bukofer en lo referente a la contribución de la música a la evolución de los sentidos del ser humano, agudizando la percepción, la sensibilidad y la admiración por el hecho sonoro. Con esto, se logran personas que se enamoren de la música y sepan apreciarla, lo cual coincide también con los principios de la educación musical enunciados por Pascual Mejía.
-Se logró corroborar que a través del "Conflicto Cognitivo" propuesto por Piaget, y desarrollado cuando se contrastan los esquemas externos con los esquemas internos, el estudiante siente la necesidad de buscar respuestas que le permitan volver al equilibrio cognitivo.

-Se presentaron casos de estudiantes con dificultades en la comprensión del proceso de lectoescritura propuesto; esto se puede explicar por cuanto entre los 15 y los 20 años de edad se efectúa la plena aparición de las operaciones formales teniendo en cuenta la riqueza de la estimulación cognitiva que alcanzó el individuo en su infancia (Clemente, 1996) lo que indica que estos estudiantes probablemente no tuvieron la oportunidad en temprana de edad de desarrollar plenamente sus capacidades cognitivas.

-El proceso diseñado y ejecutado en cada uno de los talleres que conforma esta investigación, se enfoca en la exploración del mundo sonoro externo, para lograr en cada uno de los estudiantes la conformación, construcción y comprensión del mundo sonoro interno.

-La interacción con el material diseñado en cada uno de los talleres, permitió lograr la intención del investigador de "aprender música haciendo música", lo cual coincide con lo manifestado por Pascual (2002) jugar con la música permite la construcción del conocimiento y el desarrollo del gusto por la música.

-En el desarrollo de este estudio se logró crear un ambiente de aprendizaje motivador que permitiera al estudiante la vivencia de diferentes aspectos como el canto, la ejecución de instrumentos, la audición, entre otros.

-Para el caso de los estudiantes de la Licenciatura en Educación Artística y Cultural, el estudio de los lenguajes artísticos, incluido el de la música, hace parte de su proyecto de vida el cual pretenden ofrecer al servicio de la humanidad, convirtiéndose en multiplicadores de la experiencia sonora.

\section{Referencias Bibliográficas}

Clemente, A. (1996): Psicología del Desarrollo Adulto. Narcea. Madrid Hemsy De Gainza, Violeta (2000) Educación musical en el siglo XX. Recuperado de http://www.scielo.cl/scielo.php?pid=S0716$27902004020100004 \&$ script $=$ sci_arttext

Jorquera, M. C. (s/f). La Música y la Educación musical en la Sociedad Contemporánea. Recuperado de

http://musica.rediris.es/leeme/revista/jorquera00.pdf

Martín García, A. V. (2000). Más allá de Piaget:Cognición adulta y educación. Recuperado de

http://gredos.usal_es/jspui/bitstream/10366/71861/1/Mas_alla _de_Piaget_cognicion_adulta_y_ed.pdf?origin=publication_detail

Pascual (2002). Didáctica de la Música para primaria. España: Prentice Hall.

Pozo, J. I. (1996): Maestros y aprendices. Alianza. Madrid.

Werner, H. (1957). The concept of development from a comparative and organismic point of view. Citado por D.B. HARRIS (De.): The concept of development. Minneapolis: University of Minnesota Press. 